\title{
A 256-Element Spiral CMUT Array with Integrated Analog Front End and Transmit Beamforming Circuits
}

\author{
Alessandro Stuart Savoia \\ Department of Engineering \\ Roma Tre University \\ Roma, Italy \\ alessandro.savoia@uniroma3.it
}

\author{
Enrico Boni \\ Department of Information \\ Engineering \\ University of Florence \\ Firenze, Italy \\ enrico.boni@unifi.it
}

Marco Sautto

ST Microelectronics

Agrate (MB), Italy

marco.sautto@st.com

Fabio Quaglia

ST Microelectronics

Agrate (MB), Italy

fabio.quaglia@st.com

\author{
Barbara Mauti \\ Department of Engineering \\ Roma Tre University \\ Roma, Italy \\ barbara.mauti@uniroma3.it
}

\author{
Alessandro Ramalli \\ Department of Information \\ Engineering \\ University of Florence \\ Firenze, Italy \\ alessandro.ramalli@unifi.it
}

\author{
Giulia Matrone \\ Department of Electrical, \\ Computer and Biomedical \\ Engineering \\ University of Pavia \\ Pavia, Italy \\ giulia.matrone@unipv.it
}

\author{
Giosuè Caliano \\ Department of Engineering \\ Roma Tre University \\ Roma, Italy \\ giosue.caliano@uniroma3.it
}

\author{
Luca Fanni \\ Department of Engineering \\ Roma Tre University \\ Roma, Italy \\ luc.fanni@stud.uniroma3.it
}

Federico Guanziroli

ST Microelectronics

Agrate (MB), Italy

federico.guanziroli@st.com

\author{
Alvise Bagolini \\ Fondazione Bruno Kessler \\ Povo (TN), Italy \\ bagolini@fbk.eu
}

Stefano Passi

ST Microelectronics

Agrate (MB), Italy

stefano.passi@st.com

\section{Roberto Bardelli ST Microelectronics Agrate (MB), Italy roberto.bardelli@st.com}

\author{
Pierluigi Bellutti \\ Fondazione Bruno Kessler \\ Povo (TN), Italy \\ bellutti@fbk.eu
}

\author{
Andrea Mazzanti \\ Department of Electrical, \\ Computer and Biomedical \\ Engineering \\ University of Pavia \\ Pavia, Italy \\ andrea.mazzanti@unipv.it
}

\author{
Piero Tortoli \\ Department of Information \\ Engineering \\ University of Florence \\ Firenze, Italy \\ piero.tortoli@unifi.it
}

\begin{abstract}
MEMS-based Capacitive Micromachined Ultrasonic Transducer (CMUT) technology enables the development of advanced transducer-electronics integrated multi-chip modules (MCM) for medical imaging applications. In this paper, we present the design, fabrication and characterization of a 256-element CMUT spiral array integrated with a 256-channel analog front end (AFE) ASIC featuring highvoltage unipolar pulsers, low-noise amplifiers and a programmable transmit beamformer. The spiral array, designed to perform volumetric beam steering at $7 \mathrm{MHz}$, is characterized by a density-tapered layout of $220 \mu \mathrm{m}$-wide hexagonal elements distributed over a quasi-circular area of $10 \mathrm{~mm}$ diameter. The CMUT and the AFE were co-designed, fabricated and interconnected through an acoustically optimized 3-D packaging method. The resulting MCM was electromechanically and acoustically characterized, demonstrating the potential of the proposed approach for integrated 2-D CMUT array fabrication.
\end{abstract}

Keywords-CMUT, ASIC, analog front end, spiral array,

This work was supported by the European Union through the ENIAC JU project DeNeCoR under grant agreement number 324257, and by the Italian Ministry of Education, University and Research (PRIN 2010-2011). integrated beamformer, low-noise amplifier, 3-D packaging

\section{INTRODUCTION}

The integration of MEMS-based Capacitive Micromachined Ultrasonic Transducer (CMUT) 2-D arrays for medical imaging applications and front-end electronics has been demonstrated earlier using conventional flip-chip packaging approaches [1]. Conventional flip-chip bonding methods do not offer a preferable solution for MEMS ultrasonic transducer integration due to the negative impact of the package characteristics on the acoustic performance. Moreover, such bonding methods are often not suitable for large-area dice interconnection and for wafer-level packaging. Recently, we proposed an acoustically-optimized 3-D packaging technology for the integration of CMUT arrays and Application Specific Integrated Circuits (ASIC) compatible with wafer-level packaging [2],[3]. In this work, we present the application of the recently proposed packaging method in the design, fabrication and characterization of an integrated 256- 


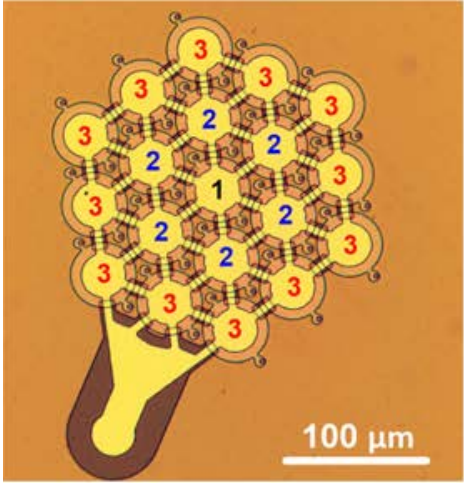

(a)

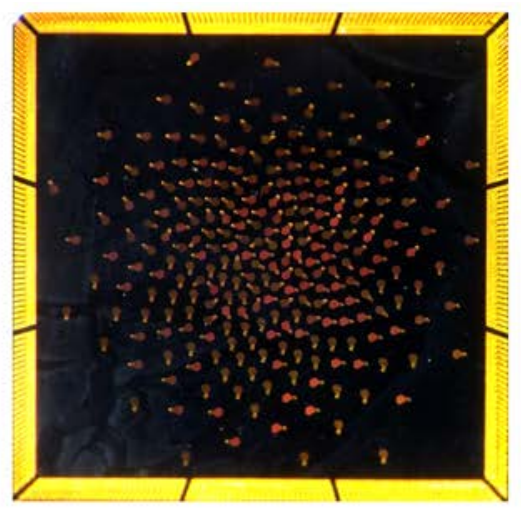

(b)

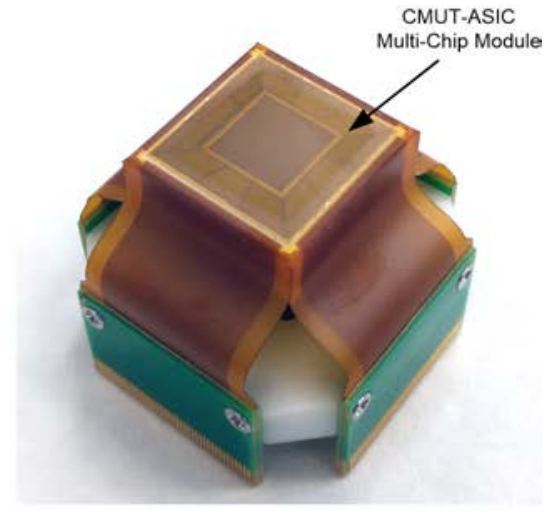

(c)

Fig. 1. Optical microscopy of a spiral array element (a) and of the CMUT spiral array (b). Photo of a packed probe head (c).

element CMUT spiral array featuring a 256-channel analog front end (AFE) ASIC. We first introduce the characteristics of the CMUT spiral array and the AFE, and the multi-chipmodule (MCM) 3-D packaging process. We then report on the electromechanical and acoustic characterization of the integrated CMUT array obtained by packing the MCM in an ultrasound probe head.

\section{INTEGRATED CMUT ARRAY}

\section{A. CMUT Spiral Array}

The CMUT spiral array layout is based on a Fermat's spiral pattern with spatial density modulation [4]. The particular spatial configuration used in the proposed array design consists in a density-tapered layout of $2561.0 \lambda$-wide elements distributed over a $45 \lambda$-wide circular area. The spatial density of the array elements is tapered using a Blackman window, which allows volumetric beam steering over a $64^{\circ}$ angular sector with a maximum one-way side lobe level (SLL) of $-20 \mathrm{~dB}$ and a maximum mainlobe level (ML) reduction of $5 \mathrm{~dB}$. The CMUT array design was dimensioned considering an operating center frequency of $7 \mathrm{MHz}$, resulting in $220 \mu \mathrm{m}$-wide elements distributed over a circular area of $10 \mathrm{~mm}$ diameter. The array fabrication was carried out using the CMUT Reverse Fabrication Process [5]. Fig. 1(a) shows a single array element composed of 19 CMUT circular cells arranged in hexagonal tiling and electrically routed to a bond pad for ASIC interconnection.

\section{B. Integrated Analog Front End}

The ASIC integrates 256 analog transceivers specifically designed around the characteristics of the CMUT spiral array. Each transceiver comprises a high voltage (HV) unipolar pulser, a T/R switch and a low-noise charge amplifier. The pulser yields pulse shaping capability provided by the possibility of controlling the amplitude, frequency, number of cycles, duty cycle, and rising/falling edges of the $(200 \mathrm{~V}$ maximum) HV pulses. The pulse shaping capability allows the reduction of the harmonic distortion introduced by the CMUT electrostatic nonlinearity [6]. The low-noise receiver is based on a capacitive-feedback transconductance amplifier topology, optimized for ultra-low-power and low-noise operation [7], [8],
[9]. The feedback capacitance can be varied by selecting four possible values, making possible the control of receive sensitivity magnitude over a $10 \mathrm{~dB}$ range. The 256 transceivers are controlled by digital circuits, which include a transmit (TX) beamformer with a time-delay resolution of $10 \mathrm{~ns}$. The ASIC is programmable through a digital interface consisting of four two-channel $100 \mathrm{MHz}$ SPIs and a daisy chain. The fabrication of the ASIC was carried out using a BCD-SOI technology by STMicroelectronics (Agrate, Italy).

\section{MCM and probe head packaging}

MCMs were obtained by processing the fabricated CMUT and ASIC wafers according to the recently proposed 3D packaging method [2], which uses $\mathrm{Cu}$ pillars and solder reflow for electrical interconnection, and patterned benzocyclobutene (BCB) for mechanical bonding. Two MCMs were assembled, i.e. a "test MCM" comprising the CMUT array and a dummy ASIC used to make the array elements directly accessible for array electromechanical and acoustic characterization, and a "fully-functional MCM" comprising both the CMUT and the programmable ASIC for TX pressure and pulse-echo testing. Each fabricated module was integrated in a probe head, following the Reverse Fabrication Packaging procedure [5], for experimental characterization. An optical microscopy of the CMUT spiral array and a photo of a packed probe head are shown in Fig. 2(b) and 2(c), respectively.

\section{CHARACTERIZATION}

\section{A. Test MCM Characterization}

Electrical impedance measurements were performed on all

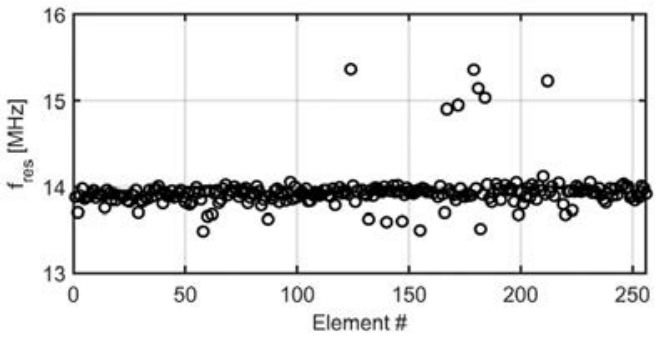

Fig. 2. Resonance frequency across the array elements. 


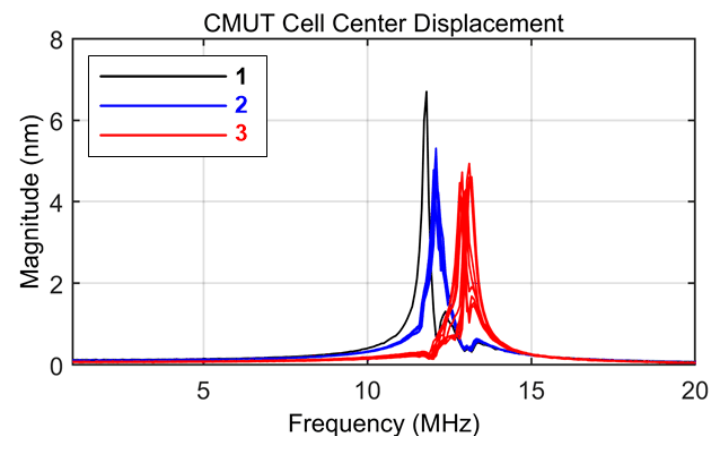

Fig. 3. Displacement spectra of the cell centers of a CMUT array element of the test MCM obtained by LDV. The legend refers to the position of the cell in the array element shown in Fig. 1(a).

the CMUT array elements in air-coupled operation using a HP4194A (Hewlett-Packard Inc., Palo Alto, CA, USA) impedance analyzer. Impedance data were processed to estimate the resonance frequency $\left(f_{\text {res }}\right)$ across the array elements (Fig. 2). The mean resonance frequency is $13.9 \mathrm{MHz}$, and the relative standard deviation is $3.2 \%$, resulting in a good uniformity of the mechanical behavior of the elements across the array. Displacement measurements were then performed on a single array element using a MSV-300 (Polytec PI Inc., Auburn, MA) laser Doppler vibrometer (LDV). Displacement spectra of the 19 cells of an array element were acquired in the 1-20 MHz range. Fig. 3 shows the displacement spectra plotted with different colors, according to the position of each cell in the array element, which is detailed in Fig. 1(a). It can be noticed that cells with equivalent positions behave similarly.

Acoustic measurements were then performed in a watertank setup. A single array element was excited using a $30 \mathrm{~V}$, 50ns unipolar pulse generated by a HP8116A (HewlettPackard Inc., Palo Alto, CA, USA), and TX pressure was acquired using a needle-type MHA9-150 hydrophone (Force Technology, Brøndby, Denmark) placed at $3 \mathrm{~mm}$ from the transducer surface. The acquired pressure signal and spectrum [Fig. 4(a)] show broadband immersion operation around 9 $\mathrm{MHz}$. Finally, the probe head was connected to the ULA-OP 256 scanner [10], [11], which was configured to drive the CMUT array elements using $7 \mathrm{MHz}, 3$-cycle, Hanning-tapered sine bursts. Radiated pressure fields were acquired at the focal depth $(20 \mathrm{~mm})$ over a $20 \times 20 \mathrm{~mm}$ area with a resolution of $0.2 \times 0.2 \mathrm{~mm}$ using a HGL-0400 (Onda Corporation, Sunnyvale, CA, USA) hydrophone in different steering conditions, i.e. $0^{\circ}$, $17^{\circ}$, and $27^{\circ}$. Fig. 5 shows the one-way beampatterns in the three steering conditions, computed as the peak negative pressure normalized to the ML in the unsteered case. Acoustic field simulations [12], [13] are reported in Fig. 5 for comparison. MLs and SLLs have been computed for both simulated and measured beampatterns. It is worth highlighting that the hydrohpone used in the experiments has a limited angular acceptance. For this reason, a rough compensation of the ML underestimation for the hydrophone directivity was carried out. Table I summarizes the results. The simulated and measured SLLs are equivalent, while the measured ML are slightly lower than the simulated ones $(5.3 \mathrm{~dB}$ for the maximum steering angle), possibly due to the inaccuracy of the applied hydrophone directivity compensation.

TABLE I. ONE-WAY BEAMPATTERNS

\begin{tabular}{|l|c|c|c|c|c|c|}
\hline \multicolumn{1}{|c|}{ Parameter } & \multicolumn{3}{c|}{ Simulations } & \multicolumn{3}{c|}{ Experiments } \\
\hline Steering angle, deg & 0 & 17 & 27 & 0 & 17 & 27 \\
\hline $\begin{array}{l}\text { Mainlobe level, dB } \\
\text { (compensated) }\end{array}$ & 0 & -2.1 & -4.7 & 0 & $\begin{array}{c}-8.3 \\
(-4.3)\end{array}$ & $\begin{array}{c}-20 \\
(-10)\end{array}$ \\
\hline Side lobe level, dB & -29.6 & -26.1 & -24.4 & -28.7 & -25 & -23.2 \\
\hline
\end{tabular}

\section{B. Fully-functional MCM Characterization}

A test board including ASIC power supply circuits was developed. A digital pattern generator (ArbStudio 1104d, LeCroy Corporation, Chestnut Ridge, NY, USA) was used to implement the programming sequences and the ASIC control signals. The probe head was connected to the test board and configured for pulse-echo operation using a single channel. The pulser was programmed for the generation of $16 \mathrm{~V}$, 4cycle, $7 \mathrm{MHz}$ square excitation signals. The probe head was immersed, and TX pressure was acquired using a needle-type MHA9-150 hydrophone placed at $3 \mathrm{~mm}$ in front of the radiating element. A stainless-steel planar reflector was then placed at the same distance in order to acquire the echo signal. Hydrophone and echo signals and the relative spectra are shown in Fig. 4(b) and 4(c), respectively, demonstrating the functionality of the MCM.

\section{Discussion AND CONCLUSIONS}

An MCM obtained by 3-D integration of a 256-element CMUT spiral array and a 256-channel analog front-end ASIC with integrated pulsers, low-noise receivers and a

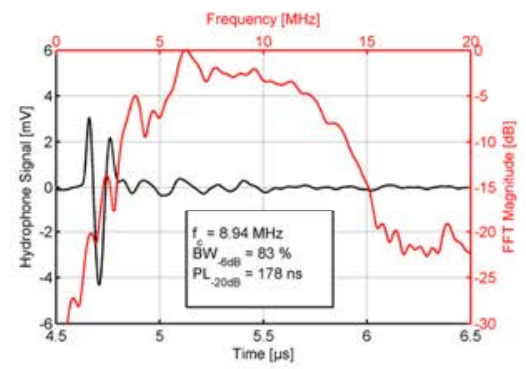

(a)

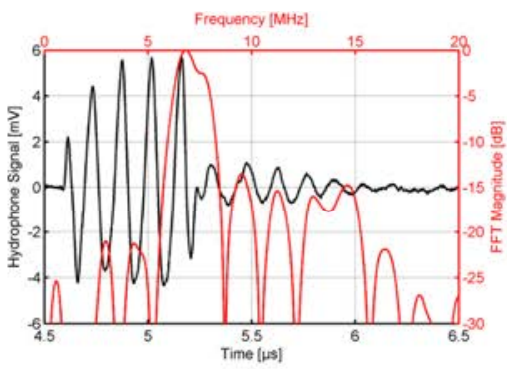

(b)

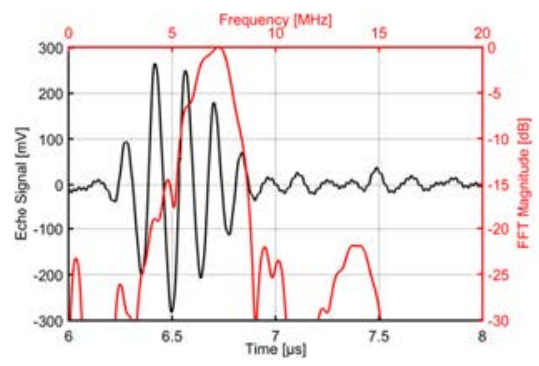

(c)

Fig. 4. Hydrophone signals: (a) TX response of one array element of the test MCM driven by an externally generated broadband pulse; (b) TX response of one array element of the fully-functional MCM driven by the ASIC. (c) Pulse-echo signal received by one array element of the fully-functional MCM. 


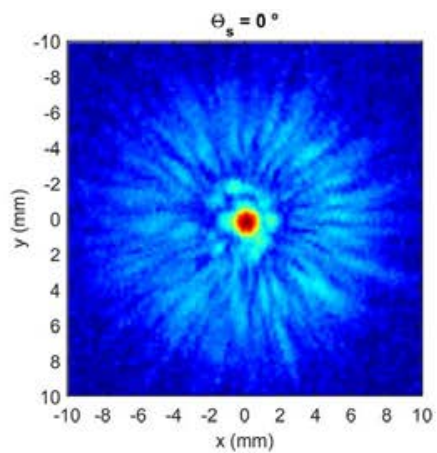

(a)

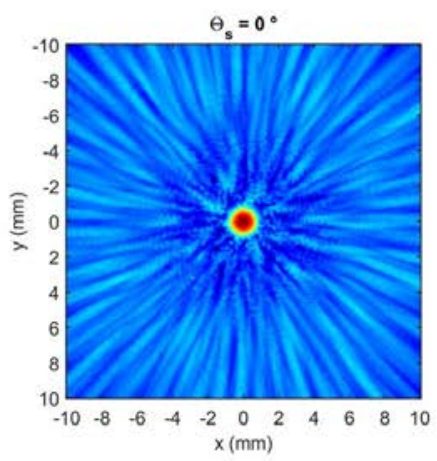

(d)

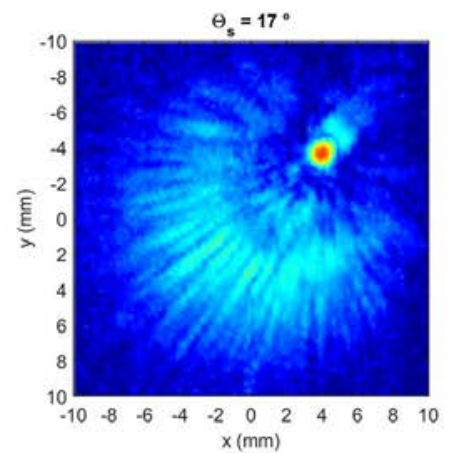

(b)

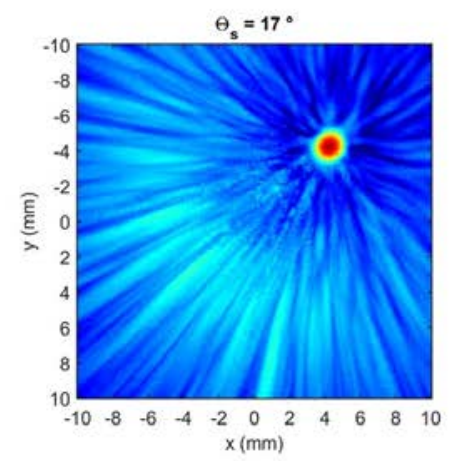

(e)

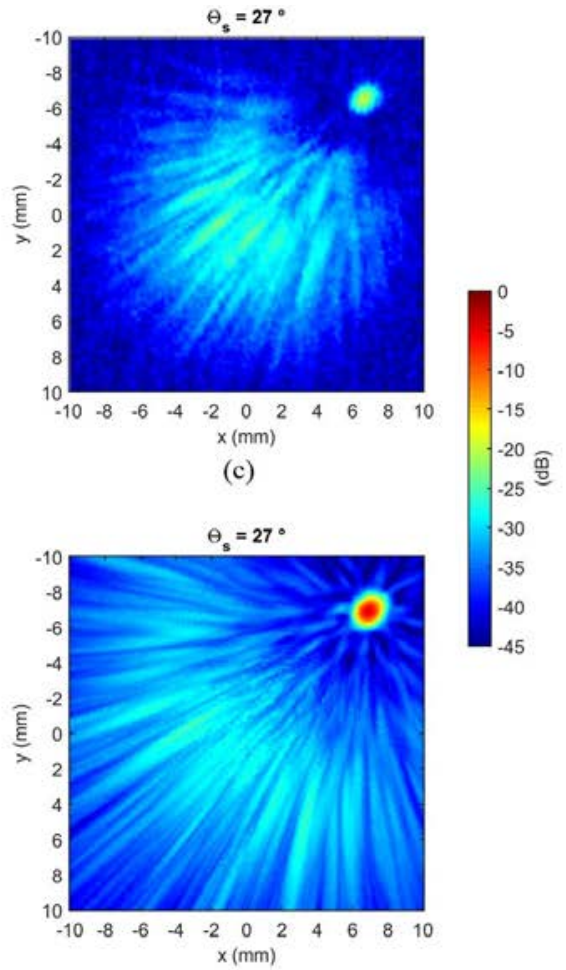

(f)

Fig. 5. Measured (a), (b), (c), and simulated (d), (e), (f), one-way beampatterns of the CMUT spiral array focused at $20 \mathrm{~mm}$ from the surface for different steering angles $\left(0^{\circ}, 17^{\circ}\right.$, and $\left.27^{\circ}\right)$. The beampatterns are normalized to the mainlobe level for the unsteered case and rendered with a dynamic range of $45 \mathrm{~dB}$.

programmable TX beamformer was successfully fabricated. Electromechanical and acoustic characterization has been performed demonstrating the functionality of both the CMUT array and the AFE ASIC. The potential of the proposed integration approach is related to its applicability to wafer-level packaging of MEMS ultrasonic transducers and ASICs. Current work is dedicated to the system integration of the probe head to perform real-time 3-D imaging experiments.

\section{ACKNOWLEDGMENT}

The authors acknowledge Dr. G. Ricotti and Dr. F. Toia for their support in the ASIC development, and Mr. E. Svaluto Moreolo for his constant and precious technical work.

\section{REFERENCES}

[1] I. O. Wygant et al., "An integrated circuit with transmit beamforming flip-chip bonded to a 2-D CMUT array for 3-D ultrasound imaging," IEEE Trans. Ultrason., Ferroelect., Freq. Contr., vol. 56, no. 10, pp. 2145-2156, 2009.

[2] A. S. Savoia et al., "A 3D packaging technology for acoustically optimized integration of 2D CMUT arrays and front end circuits," in 2017 IEEE International Ultrasonics Symposium (IUS), 2017, pp. 1-4.

[3] A. S. Savoia and G. Caliano, "MEMS-Based Transducers (CMUT) and Integrated Electronics for Medical Ultrasound Imaging," in Sensors. CNS 2016. Lecture Notes in Electrical Engineering, B. Andò, F. Baldini, C. Di Natale, G. Marrazza, and P. Siciliano, Eds. Cham: Springer International Publishing, 2018, pp. 421-429.

[4] A. Ramalli, E. Boni, A. S. Savoia, and P. Tortoli, "Density-tapered spiral arrays for ultrasound 3-D imaging," IEEE Trans. Ultrason., Ferroelect.,
Freq. Contr., Article vol. 62, no. 8, pp. 1580-1588, 2015, Art. no. 7185022.

[5] A. S. Savoia, G. Caliano, and M. Pappalardo, "A CMUT probe for medical ultrasonography: From microfabrication to system integration," IEEE Trans. Ultrason., Ferroelect., Freq. Contr., Article vol. 59, no. 6, pp. 1127-1138, 2012, Art. no. 6217561

[6] A. S. Savoia et al., "Second-harmonic reduction in CMUTs using unipolar pulsers," in Proc. IEEE Ultrason. Symp., 2015.

[7] M. Sautto et al., "A CMUT transceiver front-end with 100-V TX driver and 1-mW low-noise capacitive feedback RX amplifier in BCD-SOI technology," in Proc. Eur. Solid State Circuits Conf. (ESSCIRC), 2014, pp. 407-410.

[8] A. S. Savoia et al., "An ultra-low-power fully integrated ultrasound imaging CMUT transceiver featuring a high-voltage unipolar pulser and a low-noise charge amplifier," in Proc. IEEE Ultrason. Symp., 2014, pp. 2568-2571.

[9] M. Sautto et al., "A Comparative Analysis of CMUT Receiving Architectures for the Design Optimization of Integrated Transceiver Front Ends," IEEE Trans. Ultrason., Ferroelect., Freq. Contr., vol. 64, no. 5, pp. 826-838, 2017.

[10]E. Boni et al., "ULA-OP 256: A portable high-performance research scanner," in 2015 IEEE International Ultrasonics Symposium (IUS), 2015, pp. 1-4.

[11]E. Boni et al., "ULA-OP 256: A 256-Channel Open Scanner for Development and Real-Time Implementation of New Ultrasound Methods," IEEE Trans. Ultrason., Ferroelect., Freq. Contr., vol. 63, no. 10, pp. 1488-1495, 2016.

[12] J. A. Jensen and N. B. Svendsen, "Calculation of pressure fields from arbitrarily shaped, apodized, and excited ultrasound transducers," IEEE Trans. Ultrason., Ferroelect., Freq. Contr., vol. 39, no. 2, pp. 262-267, 1992.

[13]E. Maione et al., "PSpice modelling of ultrasound transducers: comparison of software models to experiment," IEEE Trans. Ultrason., Ferroelect., Freq. Contr., vol. 46, no. 2, pp. 399-406, 1999. 